\title{
Research Paper \\ The Relationship of Emotional Intelligence and Social Skills With Psychological Well-being in the Elderly
}

\author{
Mahnaz Akhavan Tafti $^{1},{ }^{*}$ Nahid Mofradnezhad ${ }^{1} \mathbb{C}$
}

1. Department of Educational Psychology, Faculty of Educational Sciences and Psychology, Alzahra University, Tehran, Iran.

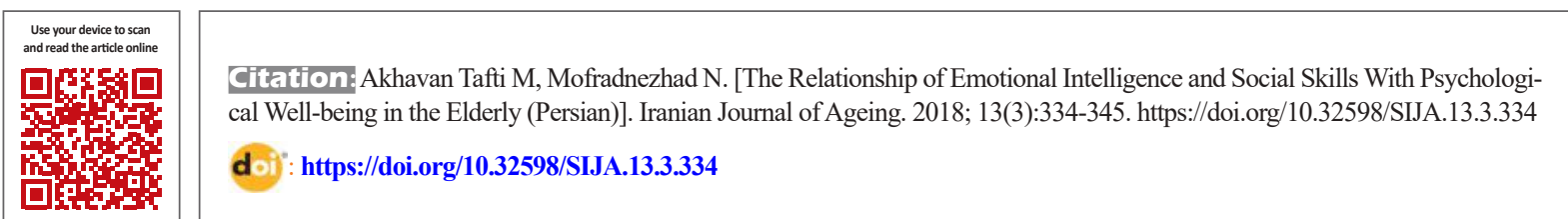

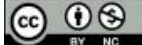

Funding: See Page 343

Received: 9 Apr 2018 Accepted: 7 Aug 2018

Keywords: Emotional intelligence, Social skills, Psychological well-being, Elderly people

\begin{abstract}
AB STRACT
Objectives Elderly People experience feelings of loneliness and isolation which is due to lack of social and emotional skills. Hence, we aimed to examine the relationship between emotional intelligence and social skills with psychological well-being in the elderly.

Methods \& Materials In this cross-sectional study, 210 elderly of Tehran City were enrolled using convenient sampling method. They completed Emotional Intelligence Inventory, Social Skill Inventory and Psychological Well-Being Inventory. Data were analyzed using Pearson and Canonical Correlation.

Results Results indicated that components of emotional intelligence and social skills were positive predictors of psychological well-being in elderly. Canonical correlation analysis showed that four fundamental variables are needed to understand the relationships between the set of criterion and predicate variables. Fundamental coefficients for the first four dimension were $0.85,0.58,0.45$ and $0.31(P<0.005)$.

Conclusion Lack of social skills and emotional intelligence are associated with low psychological wellbeing in elderly. So, training programs for improving these skills in the elderly are recommended.
\end{abstract}

\section{Extended Abstract}

\section{Objectives}

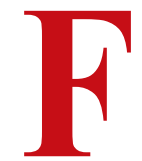

or many elderly people, the last years of life are considered to be periods of isolation and reduction of social roles when their active participation in society diminishes. These issues make the elderly feel less secure at the community level and, with increase depression, isolation and loneliness, their mental health is compromised. Due to the importance of the psychological problems and related disorders in the elderly group, more research is needed on the positive and negative factors associated with the psycho- logical health of this group so that effective interventions could be made to increase the well-being of these people, and respond to their mental and social needs in addition to their physical needs. In this regard, this study aims to investigate the relationship of emotional intelligence and social skills with psychological wellbeing in the elderly.

\section{Methods and Materials}

This is a cross-sectional correlation research. Study population consists of elderly members of the Omid Cultural Center as the only elderly culture center in Tehran, Iran. Of them, 210 elderly men and women, after providing necessary explanations for informed

\section{* Corresponding Author:}

Nahid Mofradnezhad, PhD Student

Address: Department of Educational Psychology, Faculty of Educational Sciences and Psychology, Alzahra University, Tehran, Iran.

Tel: +98 (916) 3723775

E-mail: nmofradnejad@gmail.com 
participation, were selected by convenience sampling method. This study has been approved by the Ethics Committee of Alzahra University. Inclusion criteria were having at least 60 years of age, willingness to participate and ability to answer questionnaires, while exclusion criteria were having any history of admission to psychiatric clinics or referral to a therapist due to mental disorders. The Schutte Self Report Emotional Intelligence Test (SSEIT), the social skills inventory (SSI), and Psychological Well-Being Scale (PWS) were used to collect the study data. The SSEIT is 33item measure of emotional intelligence developed by Schutte et al. [1]. They reported good construct validity of questionnaire through correlation with the five factor model of personality, and the test-retest coefficient was reported as 0.78 for two weeks. In our study, its Cronbach' $\alpha$ value was obtained as 0.78 . The SSI has 90 items with six subscales developed by Riggio [2]. He estimated its test-retest reliability and Cronbach' $\alpha$ value between 0.62-0.97. In our study, its Cronbach' $\alpha$ value was obtained as 0.82 . The PWS was presented by Ryff [3] which has 77 items and six subscales. Bayani et al. [4] in their study, while confirming its reliability and validity, reported test-retest reliability of its subscales between 0.73 and 0.87 . In our study, its Cronbach' $\alpha$ value was obtained as 0.54 .

\section{Result}

The emotional intelligence and its subscales had a significant positive correlation with psychological wellbeing. Social skills and subscales of emotional expres- sivity, emotional sensitivity, emotional control, social sensitivity and social control had a significant positive correlation with emotional intelligence, while there was a significant and negative relationship between social expressivity and psychological well-being. Canonical correlation method was used to test study hypotheses. The number of canonical dimensions needed to examine the relationship between two sets of variables was 6 , where canonical correlation coefficients of only first 4 dimensions were statistically significant. For dimension 1 , the coefficient was obtained as 0.86 , while for dimensions 2, 3, and 4 they were reported as $0.583,0.453$, and 0.302 , respectively. Table 1 shows the standardized canonical correlation coefficients for each of the four dimensions among variables predicting emotional intelligence and social skills.

Results presented in Table 1 indicates that in the first dimension, the subscale of positive relations with others had significant effect. In the second dimension, spirituality and autonomy had more effects. In the third dimension, happiness, showed more determining effect; and finally in dimension 4, happiness, satisfaction with life, autonomy and personal growth were determinants.

The first dimension suggests that a combination of emotional regulation, social control, and social sensitivity will result in less positive relations with others. The second dimension shows that a combination of emotional regulation and lower social expressivity with more emotional sensitivity will result in less positive re-

Table 1. Standardized canonical correlation coefficients for subscales of emotional intelligence and social skills

\begin{tabular}{|c|c|c|c|c|c|}
\hline \multicolumn{2}{|r|}{ Variables } & \multicolumn{4}{|c|}{ Standardized Correlation Coefficient } \\
\hline Independent & Components & Dimension 1 & Dimension 2 & Dimension 3 & Dimension 4 \\
\hline \multirow{3}{*}{$\begin{array}{l}\text { Emotional intel- } \\
\text { ligence }\end{array}$} & Appraisal and expression of emotion & -0.139 & -0.284 & -0.072 & 1.321 \\
\hline & Regulation of emotion & -0.316 & 0.536 & -0.136 & -0.26 \\
\hline & Utilization of emotion & 0.008 & 0.339 & -0.338 & -0.440 \\
\hline \multirow{6}{*}{ Social skills } & Emotional expressivity & -0.001 & -0.062 & -0.249 & -0.126 \\
\hline & Emotional sensitivity & -0.115 & 0.478 & 0.018 & -0.245 \\
\hline & Emotional control & 0.020 & 0.020 & -0.086 & 0.327 \\
\hline & Social sensitivity & -0.236 & -0.776 & 0.524 & 0.293 \\
\hline & Social Sensitivity & 0.238 & -0.205 & -0.478 & -0.156 \\
\hline & Social control & -0.443 & -0.215 & 0.679 & -0.698 \\
\hline
\end{tabular}


lations with others, and more spirituality and autonomy. According to the third canonical dimension, a combination of less emotion and more social control with higher happiness, will bring less life satisfaction, less spirituality, and more autonomy. In the fourth dimension, the combination of more emotional appraisal and less social and emotional control is significantly correlated with a combination of less happiness, less satisfaction with life, and more autonomy and personal growth.

\section{Conclusion}

As the results of canonical correlation test for two sets of predictor and criterion variables revealed, the number of canonical or hidden variables needed to explain the relationship between two predicator and criterion variables was 4 . In all these four dimensions, some subscales from both sets were involved. In other words, a combination of subscales from both sets predicted each of these dimensions. It seems that the elderly who have the ability to regulate their own emotions are those who are more compatible more hopeful and look at the bright side of life. Therefore, they have appropriate social skills to interact with others, and on the other hand, communication with others can be considered as a source of support for them in dealing with potential problems [20]. Therefore, these people have a higher level of well-being and satisfaction with life.

\section{Ethical Considerations}

\section{Compliance with ethical guidelines}

The Ethics Committee of Alzahra University has confirmed this research.

\section{Funding}

This research did not receive any specific grant from funding agencies in the public, commercial, or not-forprofit sectors.

\section{Conflict of interest}

There is no conflict of interest.

\section{Acknowledgements}

The authors would like to thank all who participated in our study. 


\title{
ارتباط هوش هيجانى و مهارتهاى اجتماعى با بهزيستى روانشناختى در سالمندان
}

مهناز اخوان تفتى'! •ناهيد مفردنراد'

ا - كروه روانشناسى تربيتى، دانشكده علوم تربيتى و روانشناسى، دانشكاه الزهراء تهران، ايران.

\begin{abstract}
حكינ

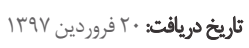

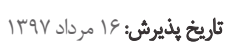

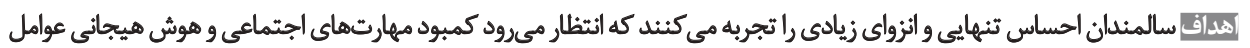

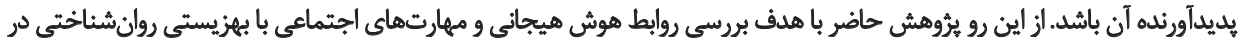
سالمندان انجام كرئت.

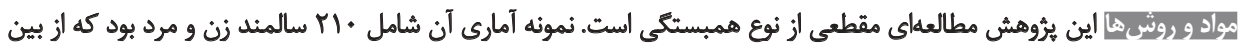

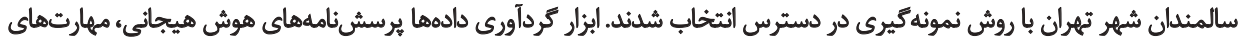

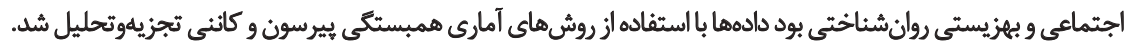

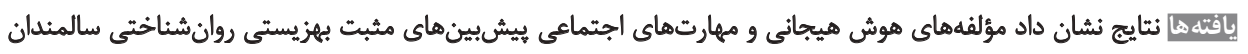

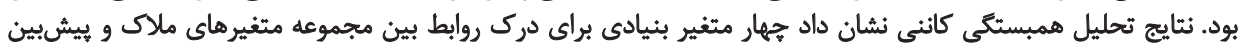

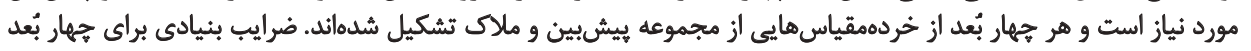

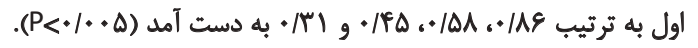

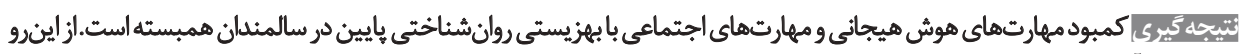

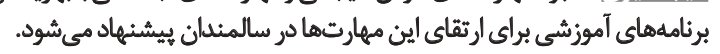

كليدوازٔهها:

هوث هيجاني،

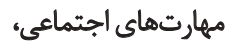
بهزيستى روانشناختي، سيالمندان
\end{abstract}

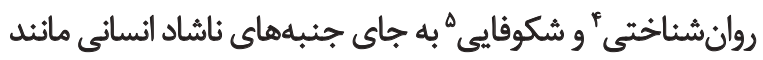

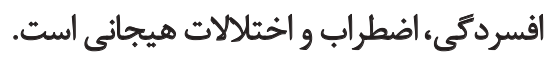

datio

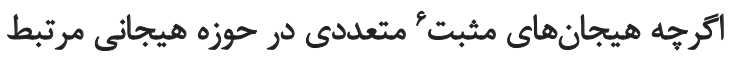

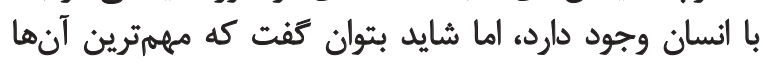

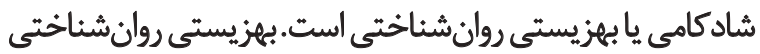

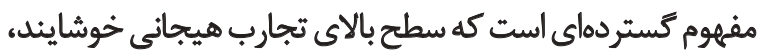

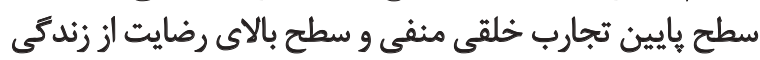

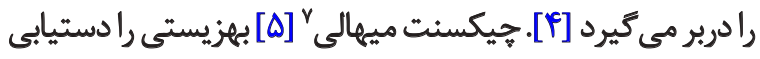

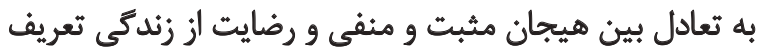

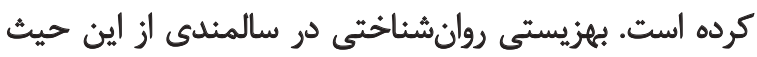

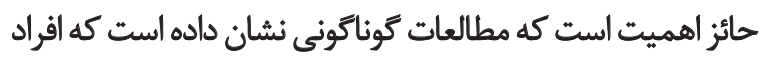
مسن آسيب يذيرى بيشترى نسبت به كاهش شادكامي مارئ دارند و

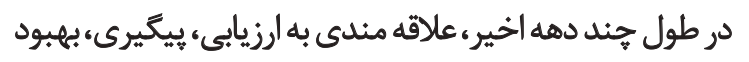

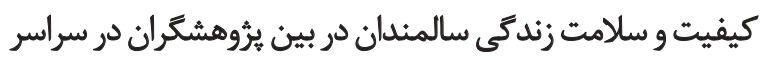

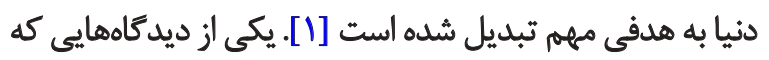

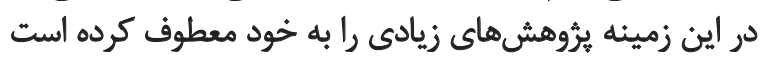

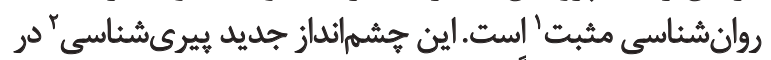

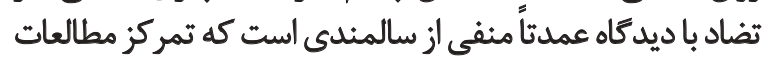

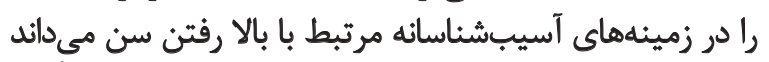

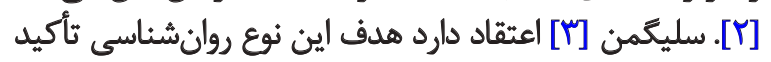

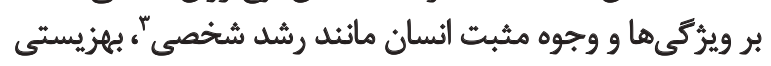

\footnotetext{
4. Psychological well-being

5. Flourishing

6. Positive emotions

7. Csikszentmihalyi
}
1. Positive psychology
2. Gerontology
3. Personal growth

* تويسئده مسئول:

ناهيل مفر دنزاد

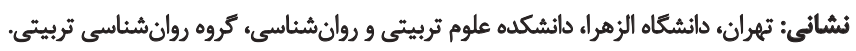
تلفن: nmofradnejad@gmail.com يست الكترونيكي: 


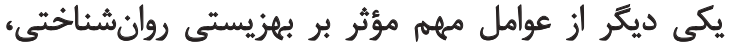

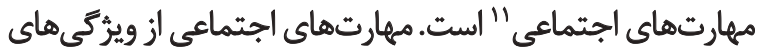

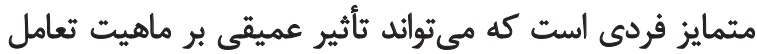

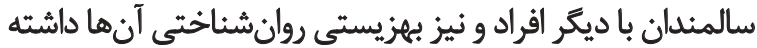

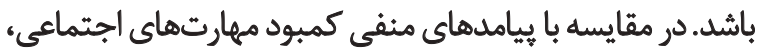

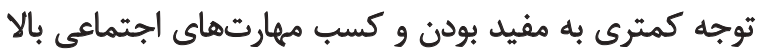

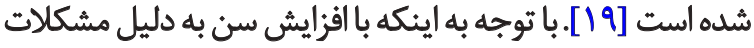

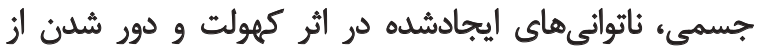

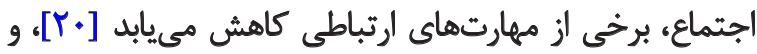

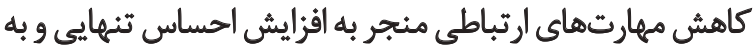

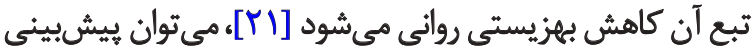

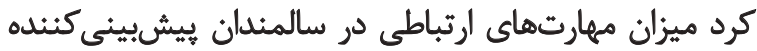

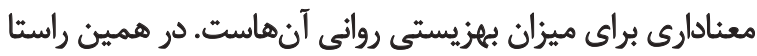

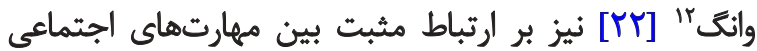

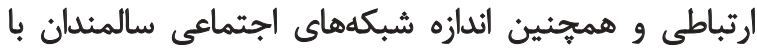
بهزيستى روائى آنها تأكيد كرده است.

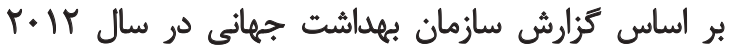

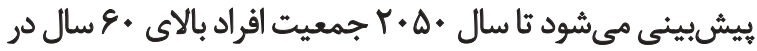

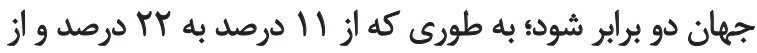

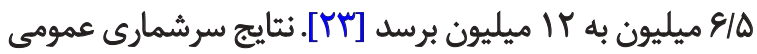

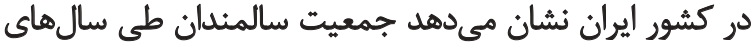
سال IFA

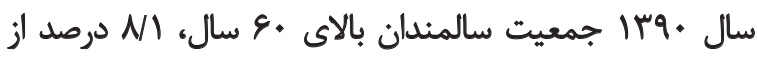

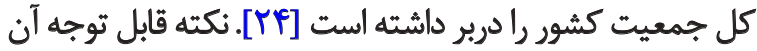

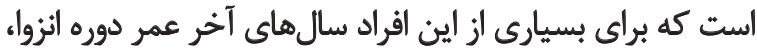

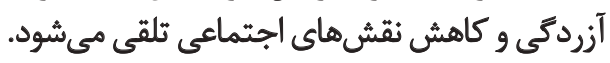
مشاركت سالمندان به عنوان قشرى سرشار از تجربه در سطح

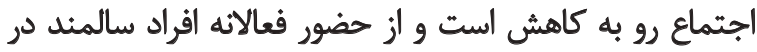

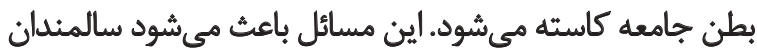

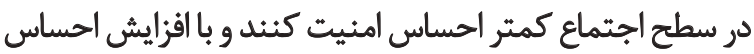

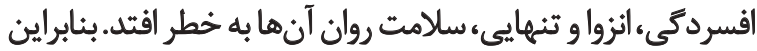

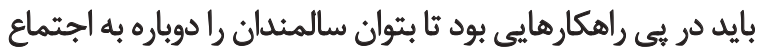

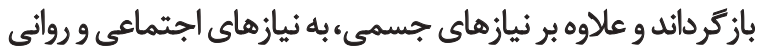
آنها نيز ياسخ كفت.

به دليل ماهيت جدى مشكلات روانى و آسيبهاى مرتبط با آن

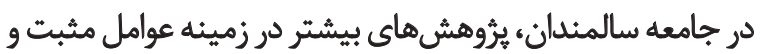

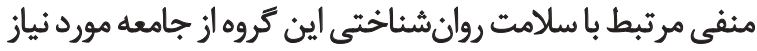

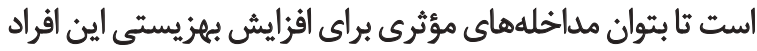

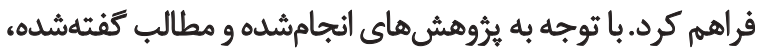

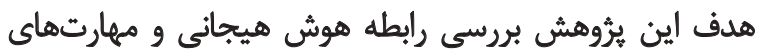

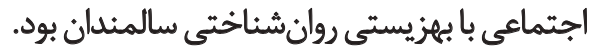

11. Social skills

12. Wang
اين امر آنها را براى انواع اختلالات هيجانى مانئد افسردگى و

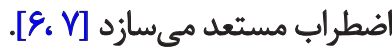

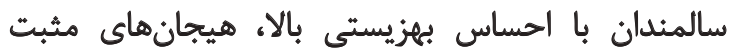

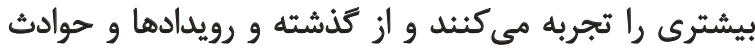

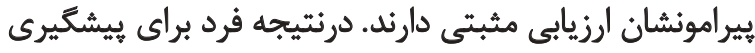

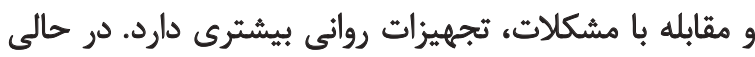

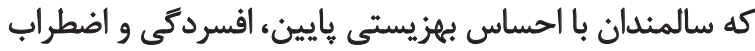

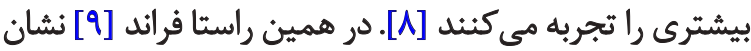

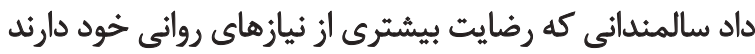

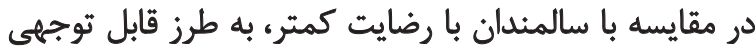

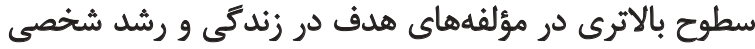

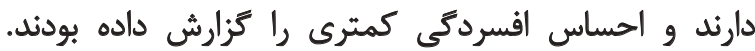

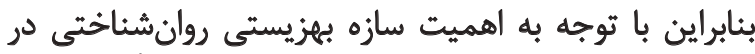

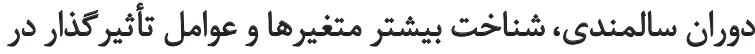
حفظ و ايجاد آن ضرورى به نظر مىرسد. بيثتر متغيرها عوان يكى از متغيرهاى مهام در ارتباط با بهزيستى روانشناختى، ماني،

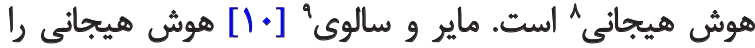

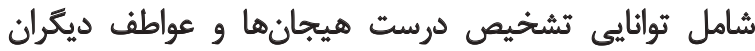

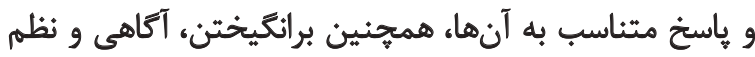

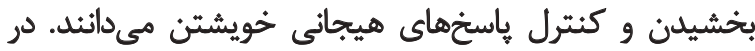

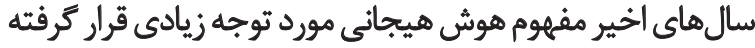

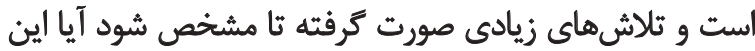

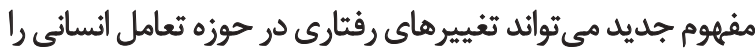
توصيف كند كه هوش و شخصيت نتوانستهاند تبيين كنند. بر اساس برخى مطالعات، مؤلفههاي هوش هيجانى بيشبين

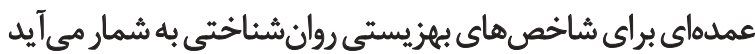

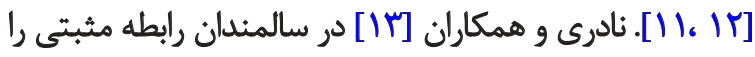

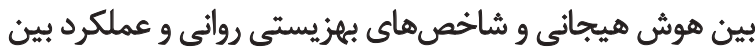

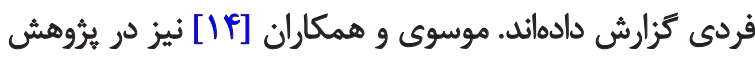

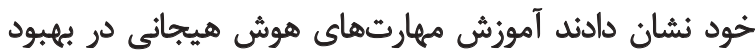

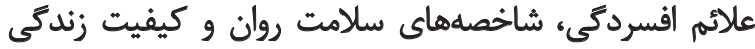

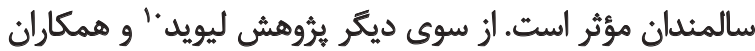

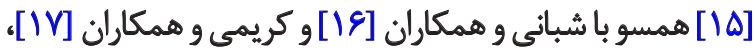

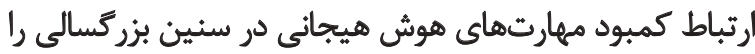

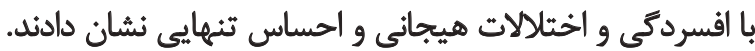

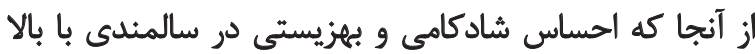
بودن سطح مهارتهاى هيجانى ارتباط دارد [1/]]، ارتباط بين

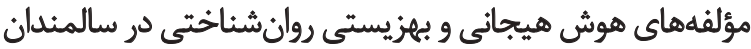
فعال در جامعه يكى از اهداف اين ثئروهش است.

8. Emotional intelligence

9. Mayer \& Salovey

10. Lioyd 


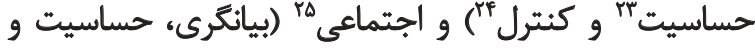

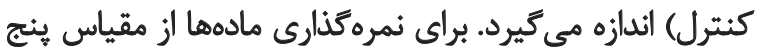

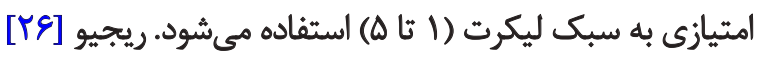

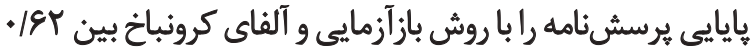

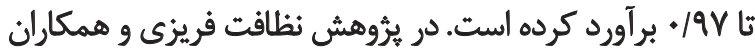

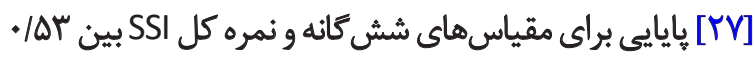

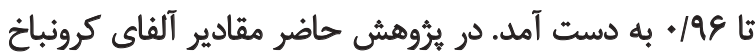

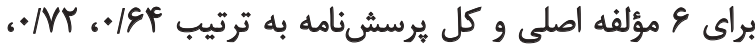

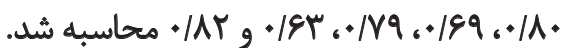

\section{مقياس بجزيستى روان شئاختى}

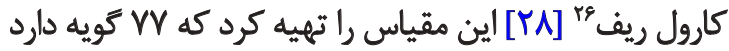

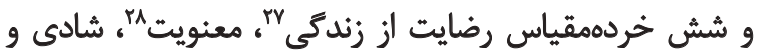

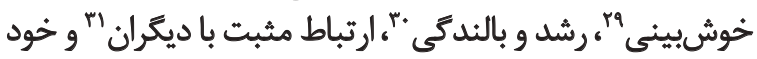

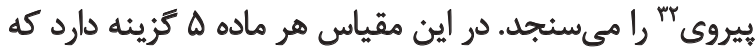

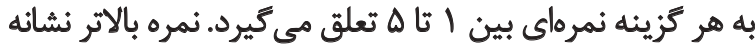

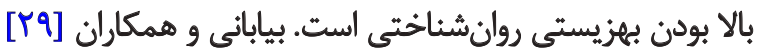

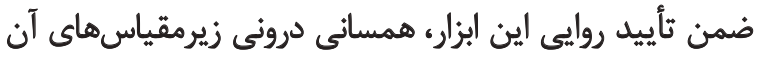

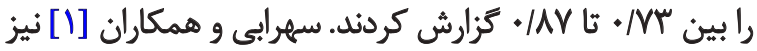

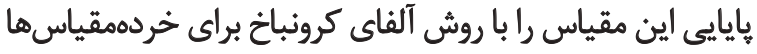

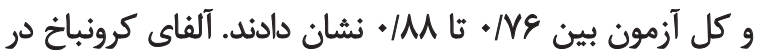

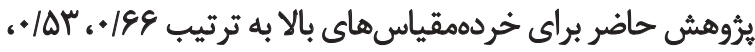

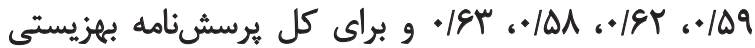

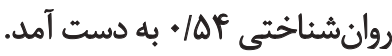

يافتهها

شاخصهاى توصيفى شامل ميانكين وانحر اف معيار متغيرهاى

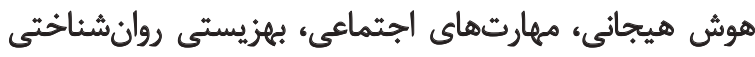

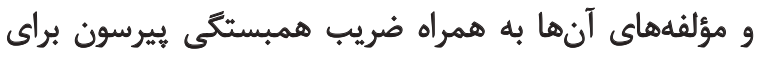

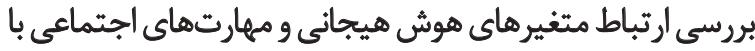
بجزيستى روانشناختى در جدول شماره إنشاني انشان داده شده است. همان طور كه مشاهده مىشود، بين هوش هيجانى و

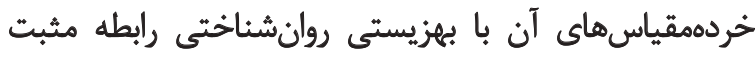

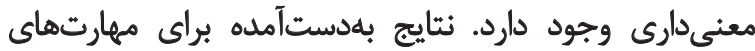

23. Sensitivity

24. Control

25. Social

26. Ryff

27. Life satisfaction

28. Spirituality

29. Happiness and optimisim

30. Maturity

31. Positive Relationship with Others

32. Self-Autonomy

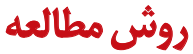

يثروهش حاضر مطالعهاي مقطعى از نوع همبستكى است. جامعه

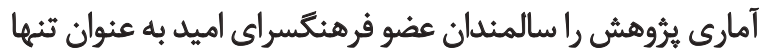

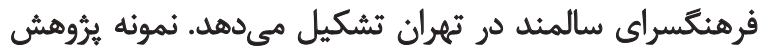

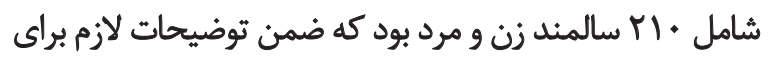

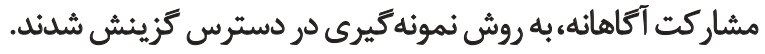

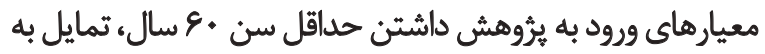

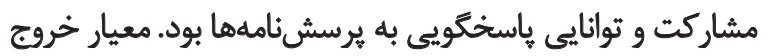

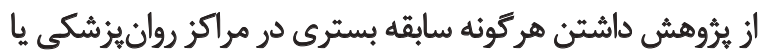

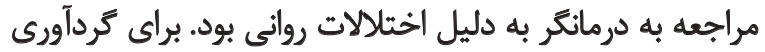

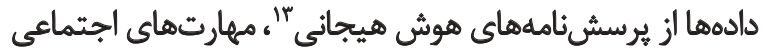

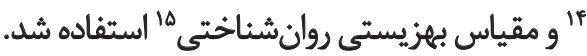

$$
\text { برسش نامه هوش هيجانى }
$$

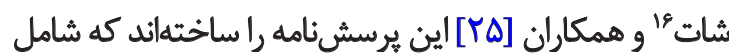

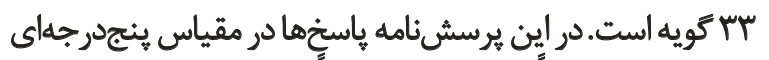

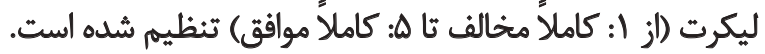

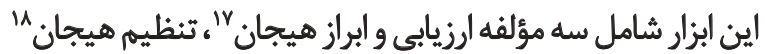

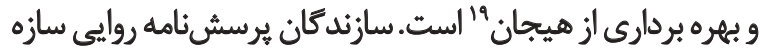

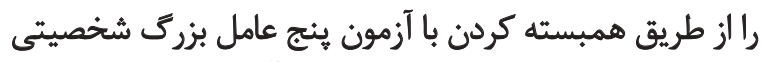

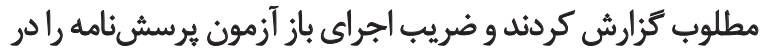

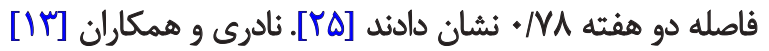

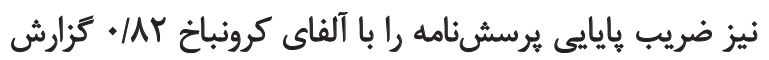

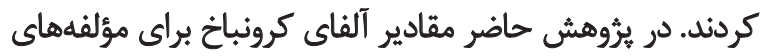

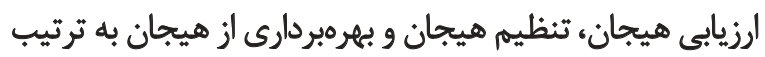

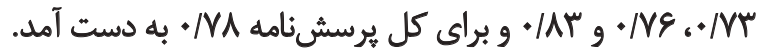

$$
\text { يرسش بامه مهارتهاى اجتماعى }
$$

ريجيو״ [عr] اين ثرسشنامه را ساخته است. يرسشنامه

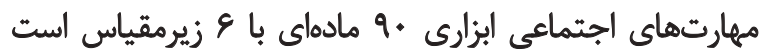

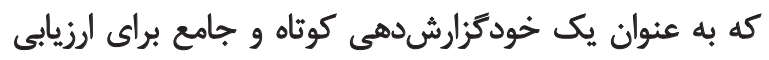

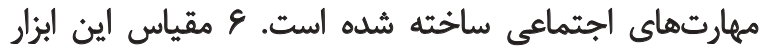

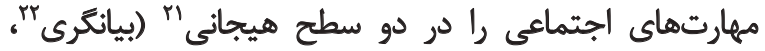

\footnotetext{
13. Emotional Intelligence Inventory (EII)

14. Social Skill Inventory (SSI)

15. Psychological Well-Being Inventory

16. Schutte

17. Emotional appraisal

18. Emotional regulation

19. Utilization of emotion

20. Riggio

21. Emotional

22. Expressivity
} 
جدول ا. ميانكين، ائحراف معيار و ضريب همبستكى متغيرهاي هوش هيجاني و مهارتهاي اجتماعى با بهزيستى روانشناختى

\begin{tabular}{|c|c|c|c|c|}
\hline سطح معنى دارى (P) & 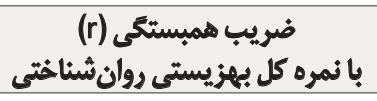 & انحراف معيار & ميانكين & متخيرها \\
\hline .10 .1 & $\cdot / \Delta Y$ & g/rq & $\Delta \cdot / \pi$ & الريابى و ابراز هيجان \\
\hline $.10+1$ &.$/ \& 8$ & $4 / \Delta 9$ & $r q / r \wedge$ & تنظيم هيجان \\
\hline$+1++1$ & $\cdot / \Delta \Delta$ & $4 / \Delta q$ & $F+/ r$ & بهرهبردارى از هيجان \\
\hline$\% 1$ &.$/ \Delta A$ & $\mid Q / A \Delta$ & ITNMF & كل هوش هيجانى \\
\hline $.1 \cdot 18$ &.$/ V$ & g/199 & eV/Na & بيانكرى هيجاني \\
\hline $.1 . .1$ &.$/ \% A$ & $N \cdot q$ & PQ/AY & حساسيت هيجائى \\
\hline $.1 .+1$ &.$/ R Y$ & NPI & peler & كثترل هيجائى \\
\hline $.1+1$ &.$- / 48$ & $q / \vartheta$ & PPIVD & بيانكّرى اجتماعى \\
\hline $.1+\cdot 1$ & $\cdot / M$ & NTq & felre & حساسيث اجتماعى \\
\hline$\bullet+1$ & .181 & NTE & $\Delta r / q e$ & كتترل اجتماعى \\
\hline \multirow[t]{8}{*}{$+1++1$} &.$/ \Delta$ & TV/AY & rAT/ga & كل مهارتهاى اجتماعى \\
\hline & & $\checkmark / \mathbb{V}$ & ENAT & رضايت از زندكى \\
\hline & & $8 / 99$ & $\Delta T / F$ & معنويت \\
\hline & & $9 / \pi 8$ & $E q / \Delta$ & شادى و هُوشينينى \\
\hline & & $r / Q V$ & rNFG & رشد و بالندكى فردى \\
\hline & & P/१ด & $r \cdot 1 \cdot r$ & ارتباط هثبت با ديكران \\
\hline & & $p / q q$ & me/gq & خودييروى \\
\hline & & MV/q & $\mathrm{rAT} / \mathrm{QA}$ & كل بهزيستى روانشناختى \\
\hline
\end{tabular}

تحليل متغيرهاى بنيادى براى تعيين تعداد ابعاد لازم براى

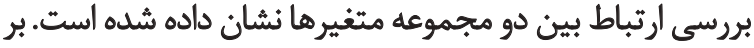

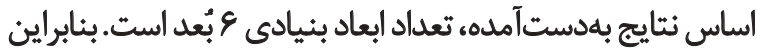

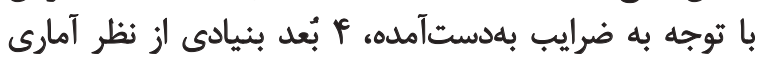

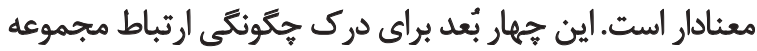
متغيرهاى بيشبين و ملاك مورد نياز است. همبستكى هاى بنيادى نيز در جدول شماره T مشاهده مي إشود.

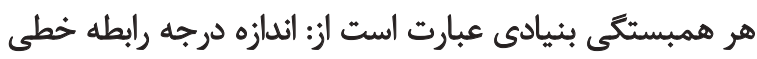

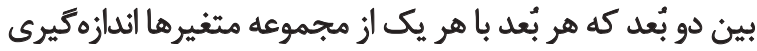

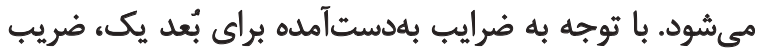

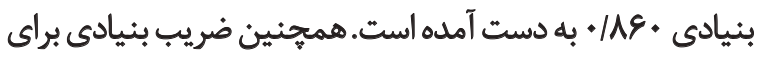

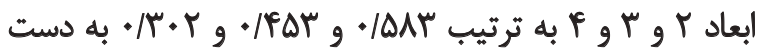

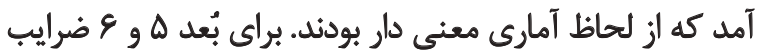
بئيادى معنادار نيستند. جدول شماره ب ضرايب استانداردشده بنيادى براى هر مُ بُعد
اجتماعى و خردهمقياسهاي بيانكرى هيجاني، حساسيت

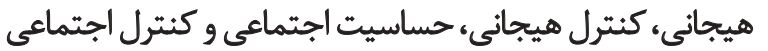

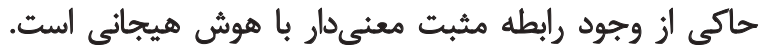

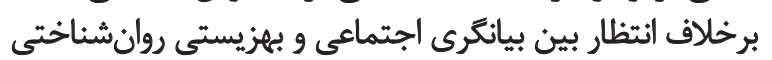
رابطه منفى معنادارى وجود دارد.

براي بررسى فرضيه يُؤهش از روش همبستكى بنيادي استفاده

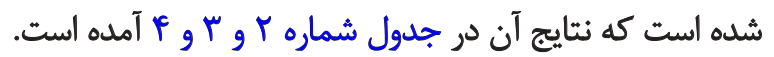

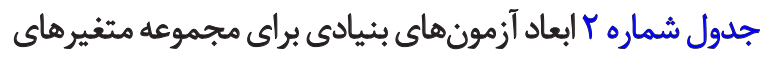

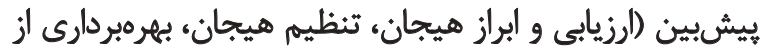

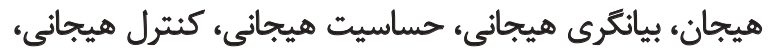

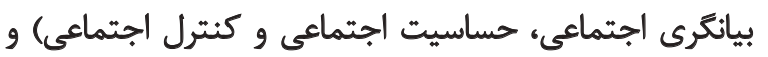

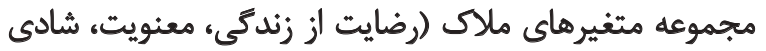

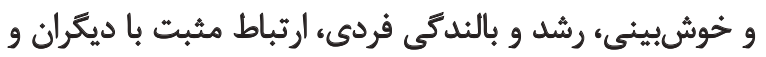

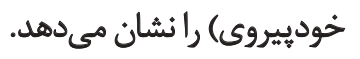
همان كونه كه در جدول شماره Y مشاهده ميشود، نتيجه 
جدول r. ابعاد آزمونهاي بنيادى براي مجموعه متغيرهاي بيشبين و ملاك

\begin{tabular}{|c|c|c|c|c|c|}
\hline احتمال & $d f_{r}$ & df, & تسبت F & همبستكى بنيادى & ابعاد \\
\hline$+1+.1$ & १Qพq. & $\Delta f$ & V/V & INE. & 1 \\
\hline $.1 . .1$ & NEV/IF & r. & $F / \mu$ & - /OAY & $r$ \\
\hline $.1 . .1$ & VIINT & TA & $r / A \Delta$ & . MAQT & $r$ \\
\hline $.1 .1 \mathrm{~V}$ & $\Delta Q / \Delta r$ & M & $1 / M^{e}$ & $\cdot r \cdot r$ & f \\
\hline . & $\mathrm{rqu}$ & 1. & I/IV & $\cdot R+r$ & $\Delta$ \\
\hline - $/ \Delta \Delta F^{\circ}$ & r.. & $p$ & - NOA & . MTY & $\varepsilon$ \\
\hline
\end{tabular}

ك

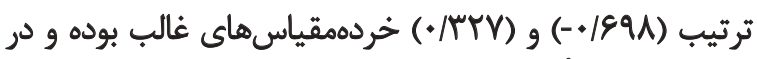
ابعاد مورد نظر ثأثير بيشترى داشتئهاند.

جدول شماره F ضرايب استانداردشده بئيادى براى هر تهمار

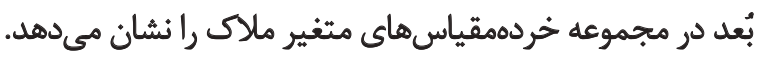

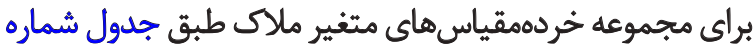

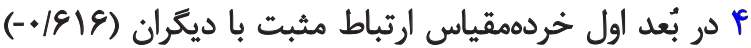

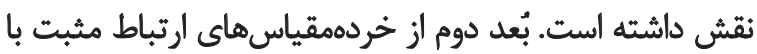

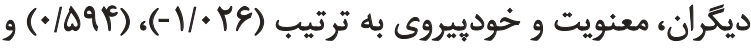

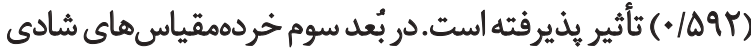

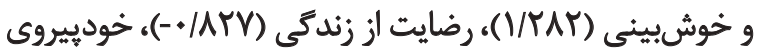
داشتهاند (19DV)

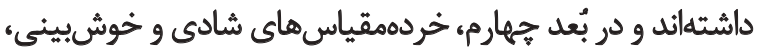

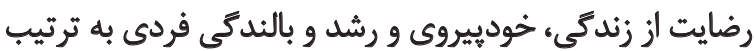

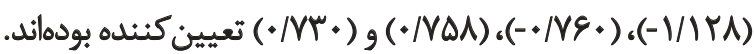

در مجموعه متغيرهاي بيشبين هوش هيجاني و مهارتهاي

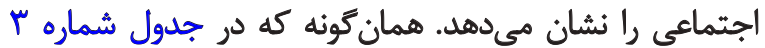

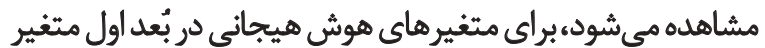

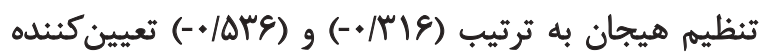

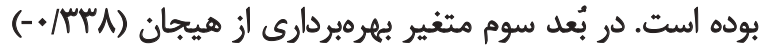

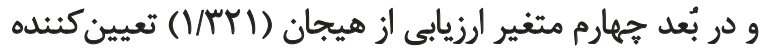

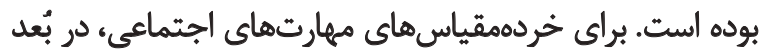

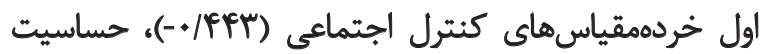

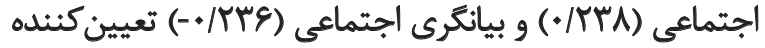

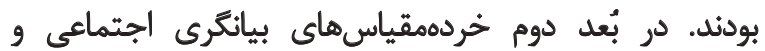
حساسيت هيجاني به ترثيب (PV\& خردهمقياس هاى كنترل اجتماعى،بيانگرى اجتماعى و حساسيت

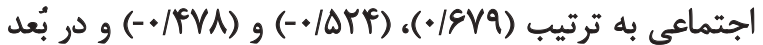

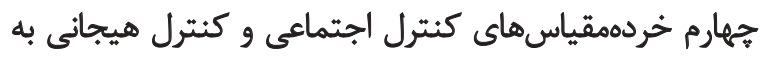

جدول لا. ضرايب بنيادى استاندارششده خردهمياس هاى هوش هيجانى و مهارتهاي اجتماعي

\begin{tabular}{|c|c|c|c|c|c|}
\hline \multicolumn{4}{|c|}{ ضريب اسيتاندارد } & \multicolumn{2}{|c|}{ متغيرها } \\
\hline نعد & بُعد & بعدد r & |بعد | & مؤلفه ها & مستقل \\
\hline I/rTI & $-* / * n$ & -+ TAF & $-* / 1 \% 9$ & ارزيايى و ابراز هيجان & \\
\hline$-\pi /$ TEA &.$- / 1 m e$ & -lare & $-* / m 18$ & تنظيم هيجان & هوش هيجانى \\
\hline.$- / 144$ & - & . /mqq & $\bullet \cdot+1$ & بهرببردارى از هيجان & \\
\hline.$- / 1 K E$ & $-\pi /$ req & -.1 .84 & $-+1+.1$ & بيانكرى هيجاني & \\
\hline.$- / M F a$ & $\cdot 1 \cdot 11$ & . /FVA &.$- / 110$ & حساسيت هيجاني & \\
\hline$\cdot / \pi T V$ & $-* / \cdot N C$ & H.r. & 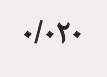 & كتترل هيجانى & مبارتهائ احتماءع \\
\hline " & $-* / \Delta M P$ & $-+/ W 9$ & - & بيانكّرى اجتماعى & \\
\hline.$- / 1 \Delta$ & $-+/ F V A$ & $-* / r \cdot \theta$ & ./MrA & حساسيت اجتماعى & \\
\hline-.184 & . 1979 &.$- /$ TIA & $-\pi / p+p$ & كنترل الجتماعى & \\
\hline
\end{tabular}

记 
جدول F. ضرايب بثيادي استانداردشده خردهمقياس هاي بهزيستى روانشئاختى

\begin{tabular}{|c|c|c|c|c|}
\hline \multicolumn{4}{|c|}{ ضرايب استاندارد } & \multirow{2}{*}{ مثغيرهاى ملك } \\
\hline بعد ع & بعد r & بعد r & بعد 1 & \\
\hline$-N Q$ &.$- / A T V$ & $\cdot / \Delta V^{p}$ &.$- / 119$ & رضايت از زندكى \\
\hline - MAD & $-+18 \cdot 8$ & .1098 & -./1rq & معنويت \\
\hline$-V / I r A$ & ITAY &.$/ T A I$ & $.10 . r$ & شادى و خوشبينى \\
\hline$\cdot M r$ &.$- / 1 Q V Y$ &.$- / 1 T f$ & $\cdot 1 *+V$ & رشد و بالندكى فردى \\
\hline Apir & $-* / 4 q 1$ & $-1 / \cdot 48$ &.$-|8| 8$ & ارثباط مثبت با ديكران \\
\hline . NOA & - IFAV & .1094 & $-\cdot /$ rVA & خودييروى \\
\hline
\end{tabular}

Lب

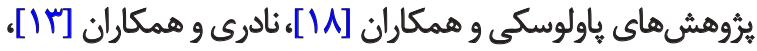

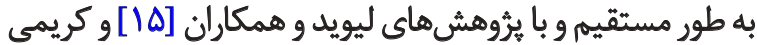
و همكاران [IV] به طور غيرمستقيم همسو است.

در نظريه هوش هيجانى، ارزيابى يعنى شناخت اساسى نظام

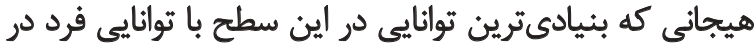

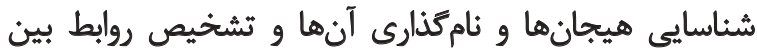

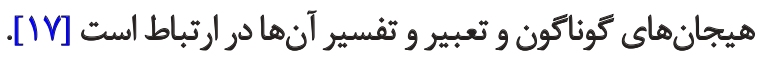

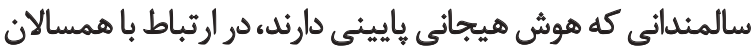

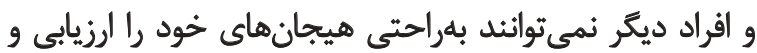

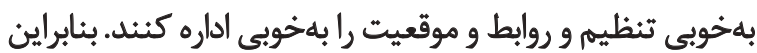

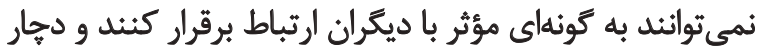

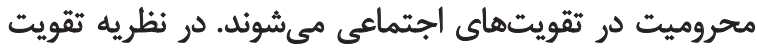

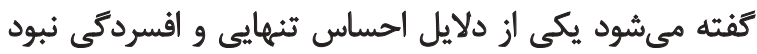

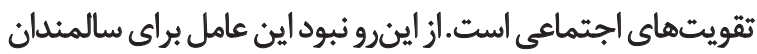

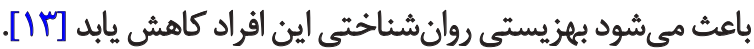
سالوى و همكاران (1999) [11] معتقدند اقرادى كه

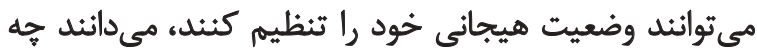

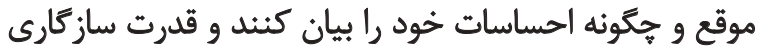

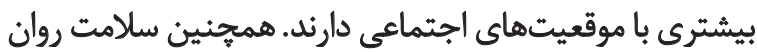

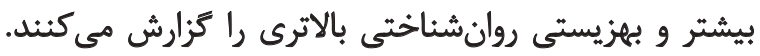

اولين بُعد يُيشنهاد مي كند كه تركيبى از تنظيمه هيجانى كمتر ،

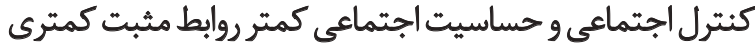

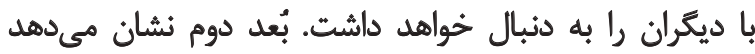

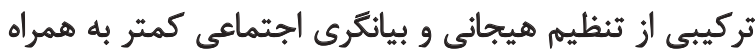

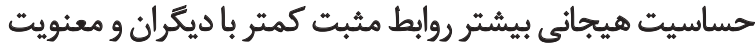

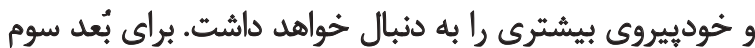

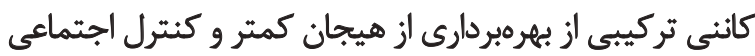

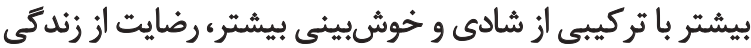

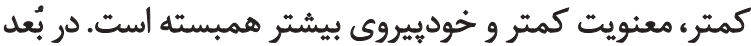

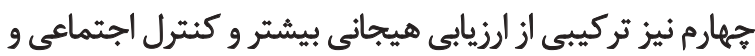

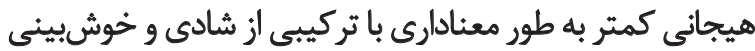

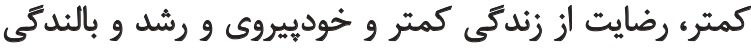

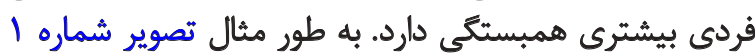

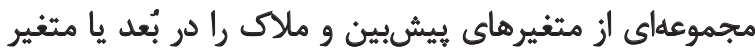

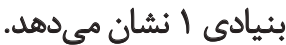
ses

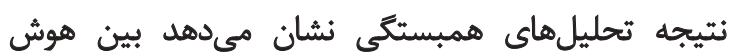

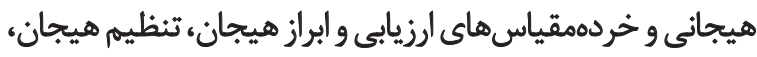

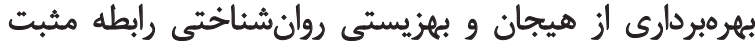

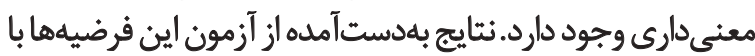

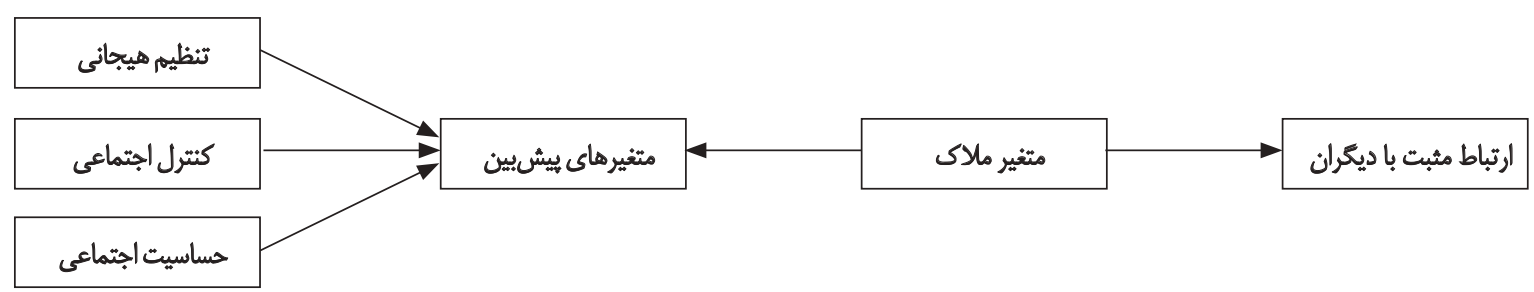




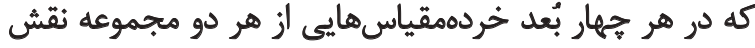

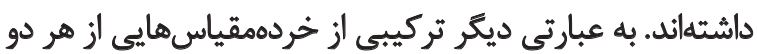

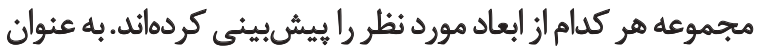

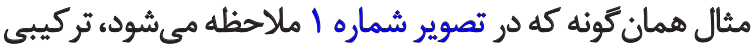

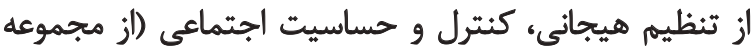

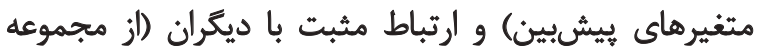

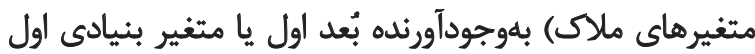

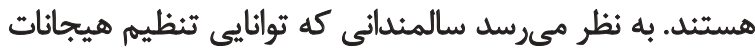

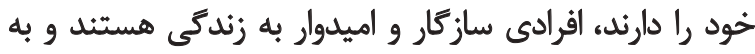

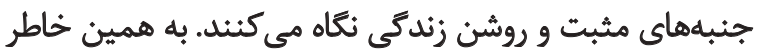

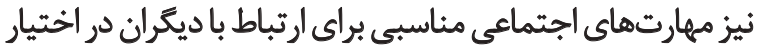

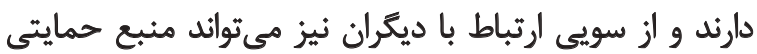

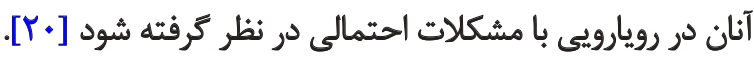
بنابراين اين افراد بهزيستى و رضايتمندى بيشترى دارئد

\section{نتيجد بيرى نهايى}

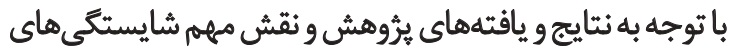

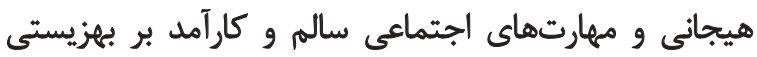

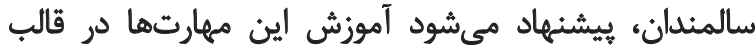

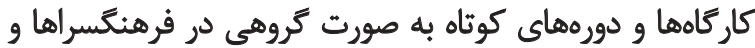

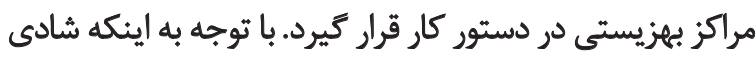

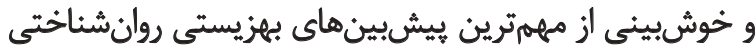

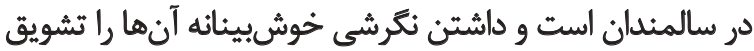
مى كند كه ارتباطات اجتماعى بيشترى با همسالان و دئن ديكران

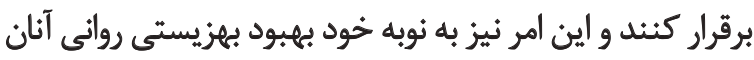

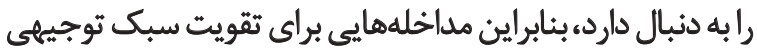

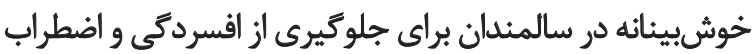
مفيد است.

ازمحدوديتهاى يروهش حاضر، جمعآورى اطلاعات بر اساس

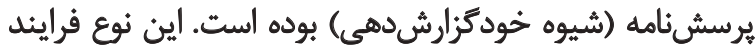

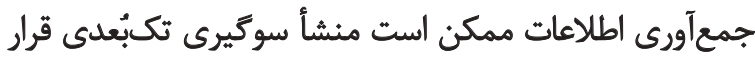

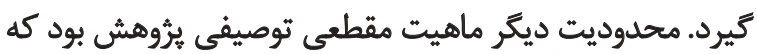

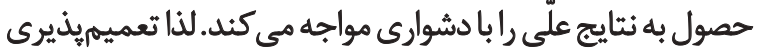

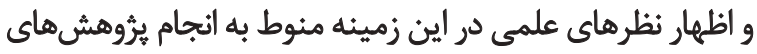

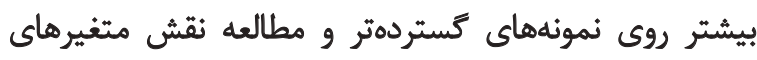
زمينهاى و واسطهاي مرتبط است.

ملاحظات اخلاقى ييروى أز اصول اخلاق يُؤهش كميته اخلاق دانشعاه الزهرا اين يثوهش را تأييد كرده است.

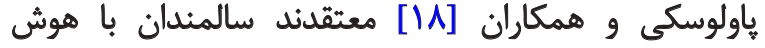

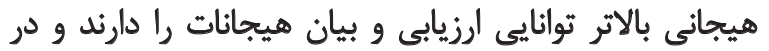

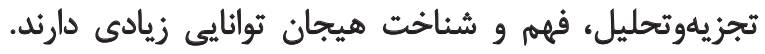

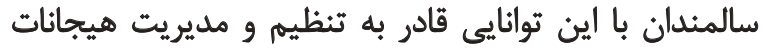

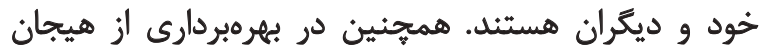

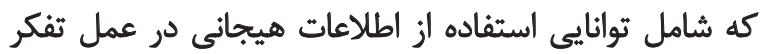

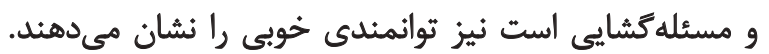

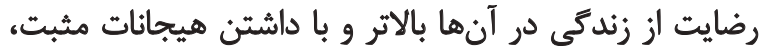

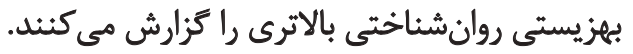

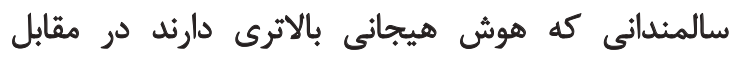

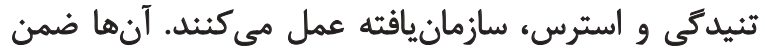

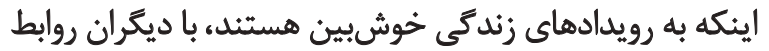

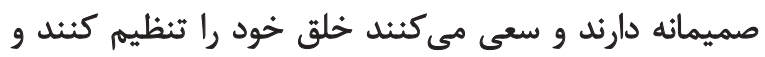

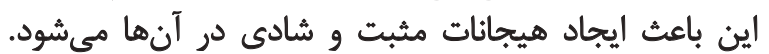

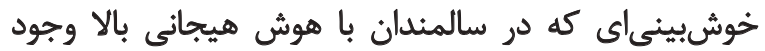

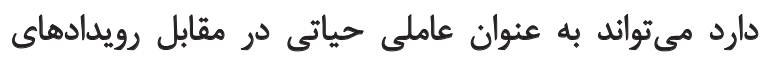

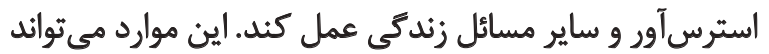

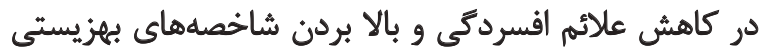

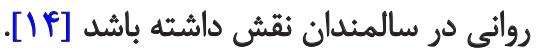

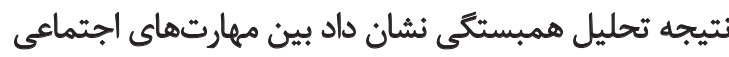

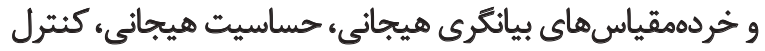

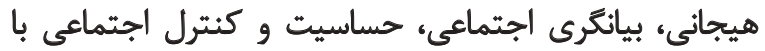

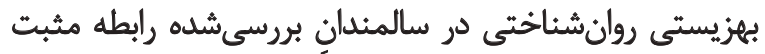

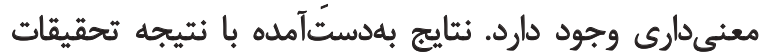

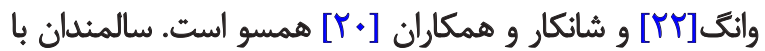

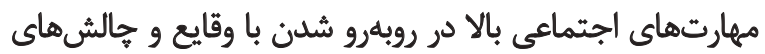

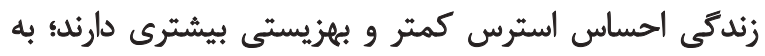

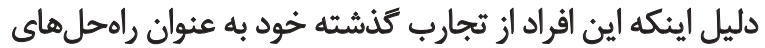

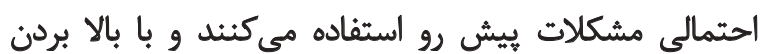

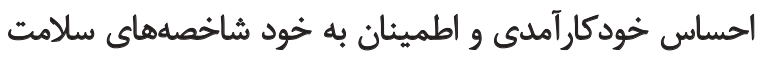
روان بالاترى رانيز نشان مى دهندا لمدين.

از سويى ديكر سالمندان به عنوان قشر آسيبذيذير در مقابل روني

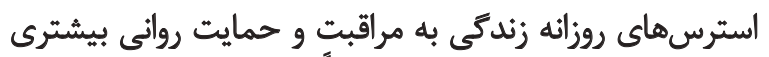

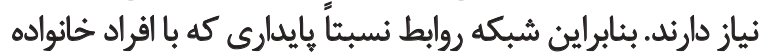

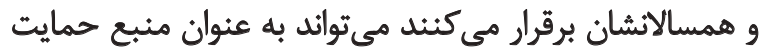

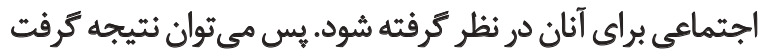

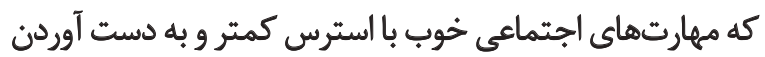

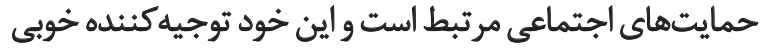

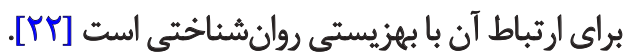

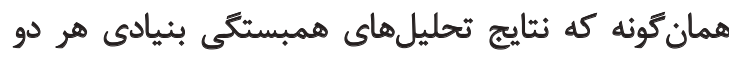

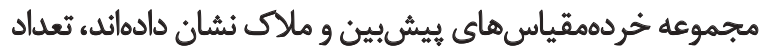

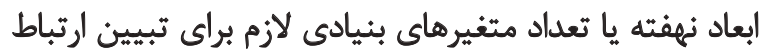

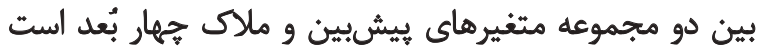




$$
\text { حامى مالى }
$$

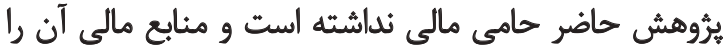
نويسندكان تأمين كرداندي

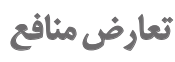

بنا بر اظهارنظر نويسندكان تعارض منافع وجود نداشته است.

$$
\text { تشكر و قدرداني }
$$

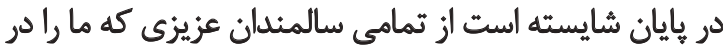
اجراى اين يُروهش يارى كردند صميمانه تشكر و قدردانى كنيه. 


\section{References}

[1] Sohrabi M, Abedanzadeh R, Shetab Boushehri N, Pasaei S, Jahanbakhsh $\mathrm{H}$. [The relationship between psychological wellbeing and mental toughness among ekders: Mediator role of physical activity (Persian)]. Iranian Journal of Ageing. 2017; 11(4):538-49.

[2] Ramirez E, Ortega A, Chamorro A, Colmenero JM. A program of positive intervention in the elderly: Memories, gratitude and forgiveness. Aginge \& Mental Health. 2014; 18(9):46370. [DOI:10.1080/13607863.2013.856858] [PMID]

[3] Seligman ME. Flourish: A visionary new understanding of happiness and well-being. NewYork: Free Press; 2011.

[4] Proyer RT, Gander F, Wellenzohn S, Ruch W. Positive psychology intervention in people aged 50-79 years: Long term effects of placebo-controlled online interventions on well-being and depression. Aginge \& Mental Health. 2014; 18(8):997-1005. [DOI:10.1080/13607863.2014.899978] [PMID]

[5] Csikszentmihalyi M, Larson R. Flow and the foundations of positive psychology. Berlin: Springer; 2014. [DOI:10.1007/97894-017-9088-8]

[6] Ferrand C, Martinent G, Durmaz N. Psychological need satisfaction and well-being in adults aged 80years and older living in residential homes: Using a self-determination theory-perspective. Journal of Aging Studies. 2014; 30:104-11. [DOI:10.1016/j.jaging.2014.04.004] [PMID]

[7] Castel A, Lluch C, Ribas J, Borràs L, Moltó E. Effects of a cognitive stimulation program on psychological well-being in a sample of elderly long-term care hospital inpatients. Aging \& Mental Health. 2017; 21(1):88-94. [DOI:10.1080/13607863.2015 1099033] [PMID]

[8] Farhadi A, Barazandeh Choqaiee S, Mokhtarpour H, Karami K, Daraeezadeh A. [Role of social support, spiritual well-being and self efficacy in predicting the subjective well-being of the elderly (Persian)]. Journal of Aging Psychology. 2015; 1(2):35-48.

[9] Waldron I, Weiss CC, Hughes ME. Interacting effects of multiple roles on women's health. Journal of Health and Social Behavior. 1998; 22:216-39.

[10] Hosseinian S, Ghasemzadeh S, Niknam M. [Prediction of quality of life in female teachers on the basis of emotional and spiritual intelligence variables (Persian)]. Quarerly Journal of Career \& Organizational Counseling. 2011; 9(3):42-60.

[11] Augusto-Landa M, Pulido-Martos M, Lopez-Zafra E. Does perceived emotional intelligence and optimism/pessimism predict psychological well-being. Journal of Happiness Studies. 2011; 12(3):463-74. [DOI:10.1007/s10902-010-9209-7]

[12] Sánchez-Álvarez N, Extremera N, Fernández-Berrocal P. The relation between emotional intelligence and subjective well-being: A meta-analytic investigation. The Journal of Positive Psychology. 2016; 11(3):276-85. [DOI:10.1080/17439760.20 15.1058968

[13] Naderi F, Asgari P, Roshani, KH, Aderyani MM. [The relationship between spiritual intelligence and emotional intelligence with satisfaction of life in elderly (Persian)]. Journal of Social Sciences. 2010; 5(13):127-38.

[14] Mousavi M, Parvin S, Farid F, Bahrainian A, Asghanehad Farid A. [Evaluation of emotional intelligence training efficiency aimed at improving the quality of life, reducing symp- toms of anxiety and depression among the elderly in Tehran nursing home (Persian)]. Journal of Fundamental of Mental Health. 2016; 18(Supl): 520-6. [DOI:10.22038/JFMH.2016.8303]

[15] Lloyd SJ, Malek-Ahmadi M, Barclay K, Fernandez MR, Chartrand MS. Emotional Intelligence (EI) as a predictor of depression status in older adults. Archives of Gerontology and Geriatrics. 2012; 55(3):570-3. [DOI:10.1016/j.archh ger.2012.06.004] [PMID]

[16] Shabani J, Hassan S, Ahmad A, Baba M. Age as moderated influence on the link of spiritual and emotional intelligence with mental health in high school students. Journal of American Science. 2010; 6(11):394-400.

[17] Karimi L, Leggat, S, Donohue L, Farrell G, Couper GE. Emotional rescue: The role of emotional intelligence and emotional labour on well-being and job-stress among community nurses. Journal of Advanced Nursing. 2014; 70(1):176-86. [DOI:10.1111/jan.12185] [PMID]

[18] Pawlowski T, Downward P, Rasciute S. Subjective wellbeing in European countries - on the age-specific impact of physical activity. European Review of Aging and Physical Activity. 2011; 8(2):93-8. [DOI:10.1007/s11556-011-0085-x]

[19] Mirdrikvand F, Panahi H, Hoseyni Ramaghani NS. [The role of communication skills, social support and disability in predicting loneliness felling of the elderly (Persian)]. Journal of Aging Psychology. 2017; 2(2):103-13.

[20] Shankar A, McMunn A, Demakakos P, Hamer M, Steptoe A. Social isolation and loneliness: Prospective associations with functional status in older adults. Health Psychology. 2017; 36(2):179-86. [DOI:10.1037/hea0000437] [PMID]

[21] Panayiotou G, Panteli M, Theodorou, M. Predictors of loneliness in a sample of college men and women in cyprus: The role of anxiety and social skills. Psychology, Community \& Health. 2016; 5(1):16-25. [DOI:10.5964/pch.v5i1.142]

[22] Wang X. Subjective well being associated with size of social network and social support of elderly. Journal of Health Psychology. 2016; 21(6):1037-42. [DOI:10.1177/1359105314544136] [PMID]

[23] Taghvaeenia A, Delavarizadeh S. [Effectiveness of training mindfulness based acceptance and commitment on body consciousness and emotional expression of the elderly (Persian)]. Journal of Aging Psychology. 2017; 2(2): 115-24.

[24] Sayadi Sarini M, Hojatkhah SM, Rashidi A. [The effectiveness of positive thinking skills on enhancement of psychological well-being and decreased of loneliness in elderly women (Persian)]. Journal of Aging Psychology. 2016; 2(1):61-71.

[25] Schutte NS, Malouff JM, Hall LE, Haggerty DJ, Cooper JT, Golden CJ, et al. Development and validation of a measure of emotional intelligence. Personality and Individual Differences. 1998; 25(2):167-77. [DOI:10.1016/S0191-8869(98)00001-4]

[26] Riggio RE. Assessment of basic social skills. Journal of Personality and Social Psychology. 1986; 51(3):649-60. [DOI:10.1037/0022-3514.51.3.649]

[27] Nezafat Ferizi J, Mashhadi A, Amin Yazdi SA, Noferesti F. [The effectiveness of short term group interpersonal Psychotherapy to symptoms of depression, emotional expressiveness, social skills and quality of life in depressed univer 